\title{
Clinical binge eating, but not uncontrolled eating, is associated with differences in executive functions: Evidence from meta-analytic findings
}

\author{
Anna Prunell-Castañé ${ }^{\text {a,b }}$, María Ángeles Jurado ${ }^{\text {a,b,c }}$, Isabel García-García a,b, " \\ ${ }^{a}$ Department of Clinical Psychology and Psychobiology, University of Barcelona, Barcelona, Spain \\ ${ }^{\mathrm{b}}$ Institut de Neurociències, Universitat de Barcelona, Barcelona, Spain \\ c Institut de Recerca Sant Joan de Déu, Barcelona, Spain
}

\section{A R T I C L E I N F O}

\section{Keywords:}

Binge eating disorder

Eating disorders

Uncontrolled eating

Executive functions

Inhibitory control

Meta-analysis

Publication bias

\begin{abstract}
A B S T R A C T
Introduction: Binge eating disorder (BED) is a common psychiatric diagnosis characterized by the presence of episodes of loss of control over food consumption. Understanding the neurocognitive factors associated with binge eating pathology might help to design clinical strategies aimed at preventing or treating BED. However, results in the field are notably heterogeneous. In the current study, we aimed to establish whether binge eating behaviors (both at a clinical and at a non-clinical level) are associated with executive functions.

Methods: We performed a pre-registered meta-analysis to examine the link between executive functions, BED, and uncontrolled eating, a psychobiological construct closely associated with binge eating behaviors. Articles were searched on PubMed and the main exclusion criteria were lack of information about participants' age or sex distribution or adiposity measurements, studies performed in older populations (age $>65$ years old) or studies including participants with purging symptoms.

Results: Relative to healthy controls, patients with BED showed lower performance in executive functions, with a small effect size. At the same time, uncontrolled eating patterns were not associated with differences in executive functions. Neither age nor body mass index (BMI) influenced these results.

Conclusions: Our findings suggest that there is no association between performance in executive functions and variations along the non-clinical spectrum of binge eating behaviors. Small deficits in executive functions, however, seem to appear in individuals showing severe binge eating symptoms, that is, individuals meeting diagnostic criteria for BED. We speculate that the close links between BED and emotional distress could partly explain these results.
\end{abstract}

\section{Introduction}

During their lifetime, around $1.9 \%$ of individuals will develop binge eating disorder (BED) (Kessler et al., 2013), the most common eating disorder among adults (Udo \& Grilo, 2018). BED is characterized by the repeated occurrence of episodes of loss of control over food consumption without purging behaviors (Diagnostic and Statistical Manual of Mental Disorders, 2013). Moreover, it has a major burden on mental and physical health, since it is commonly associated with mood disorders, such as major depressive disorder, as well as cardiometabolic problems, such as diabetes, obesity, and hypertension (de Zwaan, 2001; Udo \& Grilo, 2019). A better understanding of the neurocognitive factors associated with BED might contribute to future clinical strategies aimed at preventing or treating pathological overeating.

Executive functions are some of the most relevant neurocognitive factors. They can be defined as a group of top-down cognitive processes needed to achieve goal-directed behavior (Diamond, 2013). It is generally accepted that working memory, inhibitory control, and cognitive flexibility are core facets of executive functions (Diamond, 2013). However, executive functions can also refer to other high-order processes such as decision making, planning, or response to feedback (Sachdev et al., 2014). Some studies have reported that patients with BED show impairments in different subdomains of executive functions. For example, relative to healthy controls, individuals with BED seem to show lower performance in working memory (Eneva et al., 2017), inhibitory control (Manasse, Goldstein, et al., 2016), and decision

Abbreviations: BED, binge eating disorder; BMI, body mass index.

* Corresponding authors at: Department of Clinical Psychology and Psychobiology, University of Barcelona, Barcelona, Spain.

E-mail addresses: majurado@ub.edu (M.Á. Jurado), isabel.garciagarcia@ub.edu (I. García-García). 
making tasks (Voon et al., 2014). Along similar lines, neuroimaging studies have suggested the existence of frontostriatal alterations in BED (Kessler et al., 2016), which could relate to the cognitive difficulties observed in these patients. It has been suggested that deficits in executive function, specifically in inhibitory control, might predict poorer treatment outcomes in BED (Manasse, Espel, et al., 2016). This indicates the possibility that cognitive performance in BED might have some prognostic value. However, both behavioral and neuroimaging results in BED and compulsive eating are notably heterogeneous, which makes it difficult to conclude on the role of neurocognitive processes underlying binge eating behaviors (García-García et al., 2020; Kakoschke et al., 2019). The use of small sample sizes along with the possibly inadequate control of potential confounding effects, such as obesity, are common limitations among the studies. Moreover, as it is often the case in psychological studies, significant results might get more impact and greater visibility than negative results. For these reasons, an objective evaluation of the literature is needed in order to establish whether or not binge eating is associated with deficits in executive function.

Binge eating can occur as a continuum of increased uncontrolled eating behaviors in non-clinical populations. This notion is in line with the NIMH Research Domain Criteria (RdoC) initiative, which conceptualizes psychopathological disorders as extremes on psychobiological dimensions (Kozak \& Cuthbert, 2016). This way, BED could be regarded as the higher end of a spectrum in uncontrolled eating (Vainik et al., 2019). The concept of uncontrolled eating arises from the observation that several eating traits, such as lack of control over eating, compulsive eating, reward-related eating, or lack of satiety, are strongly correlated with each other (Vainik et al., 2015). This suggests that they might, in fact, be part of a single broader phenotype (Vainik et al., 2015, 2019). As such, here we use the term uncontrolled eating to study binge eating traits in non-clinical populations.

Previous meta-analyses have addressed differences in inhibitory control performance in obese participants with and without clinical BED (Lavagnino et al., 2016) and have examined executive functions in patients with BED (Cury et al., 2020). However, a comprehensive characterization of executive functions and binge eating in both clinical and non-clinical populations is currently missing. To address this gap, we have performed two meta-analyses. In the first one, we examined studies analyzing group differences between patients with BED and healthy controls in executive functions. This first meta-analysis adopts a 0 vs. 1 diagnostic approach to binge eating. In the second meta-analysis, we examined papers testing for the relation between uncontrolled eating patterns and performance in executive functions; following a continuous characterization of binge eating patterns in non-clinical populations. We hypothesized that, relative to healthy control participants, patients with BED would show consistent alterations in executive functions across studies. Likewise, we hypothesized that higher scores in uncontrolled eating behaviors would be correlated with lower performance in executive functions.

\section{Methods}

\subsection{Meta-analysis design}

Prior to conducting the data search, we preregistered our design and analysis plans in Open Science Framework: https://osf.io/trn4b.

We present the results of two separate meta-analyses, which were performed according to the principles of the PRISMA-Statement (Liberati et al., 2009):

(i) Meta-analysis 1: we included studies examining group differences between BED patients and healthy controls in executive functions.

(ii) Meta-analysis 2: we included studies examining the relationship between uncontrolled eating patterns and executive functions.
We included peer-reviewed studies written in English and published until May 31st 2020. APC and IGG performed the literature search independently using PubMed, and the results were cross-validated. Whenever we detected a sample overlap, we included the study with the highest sample size. We used the following keywords to perform the literature search:

a) Terms that are related to executive functions (in both meta-analyses): i.e., executive function, working memory, planning, decision making, inhibition, task-switching, cognitive control, and flexibility.

b) Binge eating disorder (meta-analysis 1).

c) Terms that are related to uncontrolled eating (meta-analysis 2): i.e., uncontrolled eating, binge eating, disinhibited eating, emotional eating, external eating, food addiction, food craving, hedonic hunger, loss of control over eating, and compulsive eating (Vainik et al., 2015).

\section{Exclusion criteria were:}

a. Studies that do not report adiposity measurements (such as body mass index (BMI), waist circumference, or body fat percentage). We did so since obesity might be a major confound on neurocognitive studies of BED. For instance, individuals with obesity show a high prevalence of binge eating symptoms (de Zwaan, 2001), and previous studies have related obesity with subtle differences in executive function performance (Yang et al., 2018).

b. Studies that do not report participants' age or sex distribution, and studies performed in older populations (age $>65$ years old), in order to avoid age-related effects.

c. Studies including participants with recurrent purging symptoms, which might be indicative of bulimia nervosa.

Fig. 1 presents an overview of the data selection process. We included the following variables in the meta-analyses:

a) Meta-analysis 1: we collected average scores and standard deviations in executive functions, as well as sample size in participants with BED and in control participants and we calculated their effect size (Cohen's d). If a study reported more than one score in executive functions, we calculated the average effect size of all its scores.

b) Meta-analysis 2: we collected correlation coefficients (bivariate and partial) and sample size. Some papers reported correlations between executive functions and several variables reflecting uncontrolled eating. When this was the case, an average of all correlation values was entered in the meta-analysis.

Negative effect sizes reflect (a) a worse performance in patients with BED relative to control participants (meta-analysis 1), and (b) that high scores in uncontrolled eating are associated with lower performance in executive functions (meta-analysis 2). We inverted the effect size of those measures for which higher scores denote lower cognitive performance (e.g., in the case of perseverative errors of the Wisconsin Card Sorting Test or B-A scores of the Trail Making Test).

We performed the analyses using Meta-Essentials (Suurmond et al., 2017). First, we entered effect sizes into the meta-analyses using a random effects model and calculated an overall effect size for all the studies. The average effect size was calculated for those studies reporting more than one effect size. Heterogeneity across studies was examined using the index $\mathrm{I}^{2}$, which calculates the percentage of variation across studies that is an effect of heterogeneity rather than chance (Higgins et al., 2003). Rough thresholds for the interpretation are suggested to be around $25 \%$ for low, $50 \%$ for moderate, and $75 \%$ for high heterogeneity (Higgins et al., 2003). We also tested for the possible presence of publication bias by examining the funnel plot and Egger test. Using moderator analyses, we also tested for the possible effects of age and BMI on the results. 


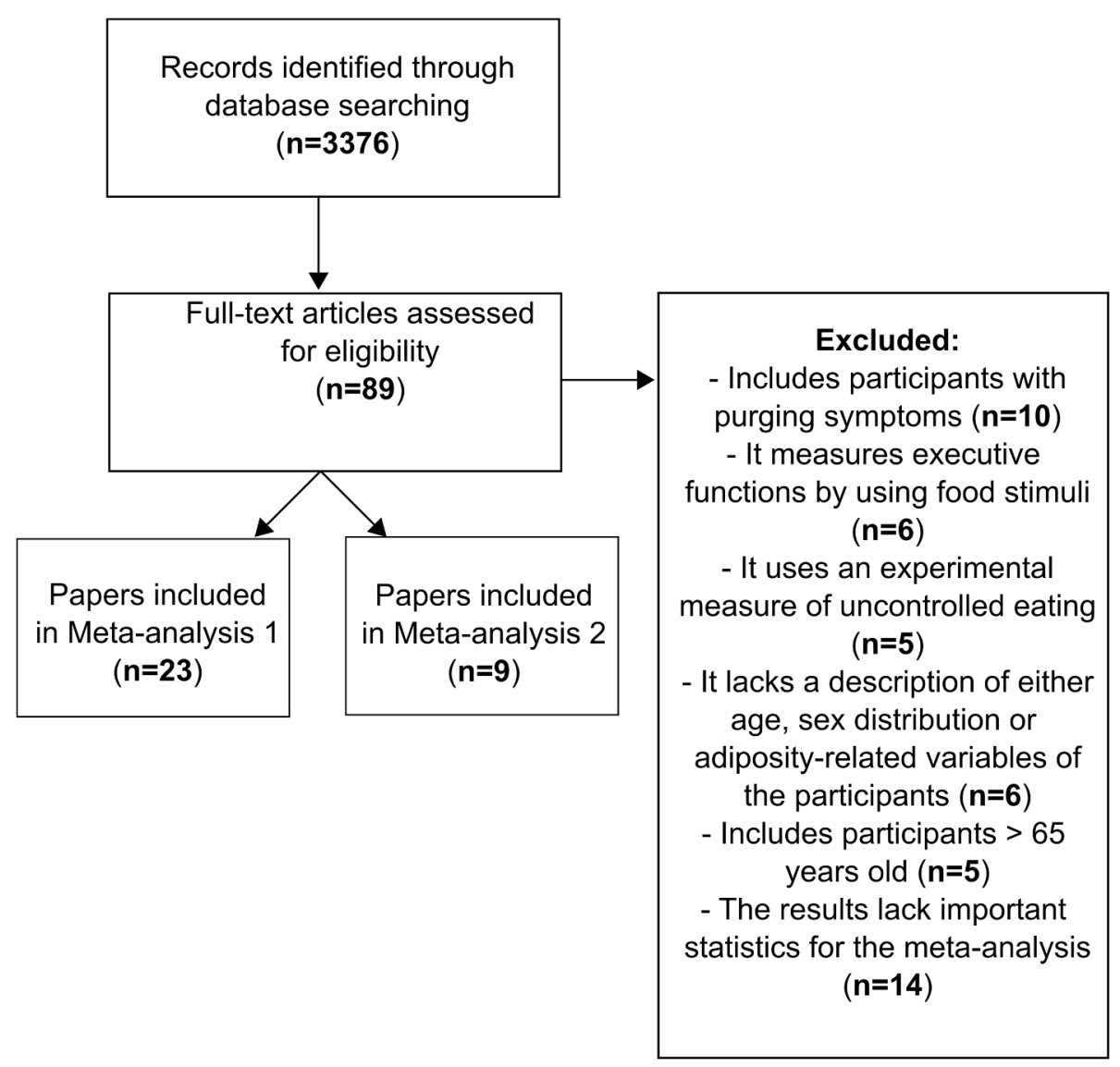

Fig. 1. Flowchart of the selection criteria for the present study.

The three main subdomains of executive functions were examined separately: (i) inhibitory control, (ii) working memory, and (iii) cognitive flexibility (Diamond, 2013). To do so, we performed additional submeta-analyses whenever the number of studies available for each of these subdomains was large enough. The minimum number of studies set to perform these sub-meta-analyses was 10 , following the recommendations from the Cochrane handbook for meta-analysis (https://tr aining.cochrane.org/handbook/current/chapter-10).

Results were considered significant if $\mathrm{p}<0.05$ after applying Bonferroni correction for each of the analyses performed using the same pool of studies.

\subsection{Deviations from the pre-registration protocol}

The pre-registration specified that we would exclude participants with neuropsychiatric diseases other than BED. This criterion was difficult to meet due to the high comorbidity that BED entails. Moreover, we had the feeling that those studies reporting neuropsychiatric comorbidities in some patients were also the ones that were particularly thorough in screening and reporting participants' clinical characteristics. For these reasons, we decided to include in the meta-analyses papers reporting psychiatric comorbidities. A systematic description of the comorbidities is included in the results. Additionally, we decided to exclude scores from tests using food stimuli to examine performance in executive functions (i.e., food-related versions of the Stroop task or the Go/No-Go test). We did so in order to avoid the possible confounding effects that food processing might involve in executive function performance. A similar approach has been followed somewhere else (Emery \& Levine, 2017).

\section{Results}

3.1. Meta-analysis 1: Executive functions in patients with binge eating disorder versus controls

We included 23 studies with $\mathrm{n}=1742$ participants (703 patients with BED and 1039 control participants). The statistical power of this meta-analysis was $90 \%$ (Quintana, 2017). A detailed description of the studies is provided in Table 1 . Supplementary Table 1 provides a description of the main tasks and questionnaires used in the included studies. The combined effect size $(\mathrm{d}=-0.22$, corrected $\mathrm{P}=0.005)$ indicated that individuals with BED showed lower performance on executive functions than control subjects. The effect size obtained was small (Fig. 2). The heterogeneity value was $I^{2}=35.63 \%$, suggesting a moderate heterogeneity across studies. A funnel plot and Egger test did not provide evidence for publication bias $(\mathrm{t}=-1.26, \mathrm{P}=0.22)$. One missing study was detected using the trim-and-fill method (Fig. 3). Moderator analysis indicated that neither age $(\beta=0.2, \mathrm{P}>0.9)$ nor BMI ( $\beta=-0.14, \mathrm{P}>0.9$ ) were associated with the relationship between binge eating and executive functions.

Two additional meta-analyses were performed focusing on the tests reflecting inhibition and cognitive flexibility. None of them showed statistically significant differences between BED patients and control participants. Results are shown in Table 2.

\subsection{Meta-analysis 2: Executive functions and uncontrolled eating in non- clinical populations}

We included 9 studies with $\mathrm{n}=889$ participants. A detailed description of the studies is provided in Table 3. One study presented separated results for normal-weight and obese participants, which were 
Table 1

Description of studies included in the first meta-analysis

\begin{tabular}{|c|c|c|c|c|c|c|c|c|}
\hline \multirow[t]{2}{*}{ Study } & \multirow{2}{*}{$\begin{array}{l}\text { BED n } \\
\text { (females) }\end{array}$} & \multirow{2}{*}{$\begin{array}{l}\text { Controls } \mathrm{n} \\
\text { (females) }\end{array}$} & \multirow[t]{2}{*}{ Pooled age } & \multirow{2}{*}{$\begin{array}{l}\text { Pooled } \\
\text { BMI }\end{array}$} & \multicolumn{2}{|c|}{ Evaluation of psychological comorbidities } & \multirow[b]{2}{*}{ Current psychiatric comorbidities } & \multirow[t]{2}{*}{ Outcomes• } \\
\hline & & & & & Scale ${ }^{\boldsymbol{\Lambda}}$ & Reported affective symptoms & & \\
\hline Aloi et al., 2015 & $45(45)$ & $45(45)$ & 28.1 & 27.7 & BDI-II & $\begin{array}{l}\text { S-Diff in depression symptoms between groups. BDI } \\
\text { score included as covariate. Depression negatively } \\
\text { correlated with cognitive flexibility }\end{array}$ & - & $\begin{array}{l}\text { Cognitive Flexibility: WCST (perseverative errors), } \\
\text { TMT (B-A). } \\
\text { Inhibition: HSCT(B-A) } \\
\text { Others: IGT (total net score), RCFT (accuracy) }\end{array}$ \\
\hline $\begin{array}{l}\text { Banca et al., } \\
2016\end{array}$ & $32(18)$ & $126(60)$ & 43.22 & 25.38 & BDI-II & $\begin{array}{l}\text { S-Diff in depression between groups. Results tested } \\
\text { before and after covarying for depression (no } \\
\text { differences found) }\end{array}$ & - & $\begin{array}{l}\text { Cognitive Flexibility: Probabilistic Reversal Task ( } \mathrm{n}^{\circ} \\
\text { of trials to criterion of } 4 \text { correct sequential choices, } \\
\text { reward condition in acquisition phase) }\end{array}$ \\
\hline $\begin{array}{l}\text { Bartholdy et al., } \\
2017\end{array}$ & $11(11)$ & $28(28)$ & 25.79 & 23.96 & DASS-21 & $\begin{array}{l}\text { NS-Diff in depression between groups. Depression } \\
\text { was not correlated with monetary temporal } \\
\text { discounting task }\end{array}$ & $\begin{array}{l}\text { BED group: psychotropic medication } \\
(\mathrm{n}=1)\end{array}$ & $\begin{array}{l}\text { Others: Monetary temporal discounting task (area } \\
\text { under curve) }\end{array}$ \\
\hline $\begin{array}{l}\text { Blume et al., } \\
2018\end{array}$ & $42(30)$ & $23(17)$ & 38.95 & 42.35 & PHQ-9 & $\begin{array}{l}\text { S-Diff in depression symptoms between groups. No } \\
\text { further control }\end{array}$ & - & $\begin{array}{l}\text { Cognitive Flexibility: WCST (perseverative errors) } \\
\text { Inhibition: GNG (commission errors) } \\
\text { Others: IGT (total net score), DDT (area under } \\
\text { curve) }\end{array}$ \\
\hline $\begin{array}{l}\text { Córdova et al., } \\
2017\end{array}$ & $18(12)$ & $18(15)$ & 45.1 & 38.54 & - & - & - & Others: IGT (total net score) \\
\hline $\begin{array}{l}\text { Danner et al., } \\
2012\end{array}$ & $19(19 x$ & $48(48)$ & 38.94 & 29.26 & IDS-SR & $\begin{array}{l}\text { S-Diff in depression symptoms between groups. } \\
\text { Depression as covariate had no effect on IGT } \\
\text { performance }\end{array}$ & - & Others: IGT (total net score) \\
\hline $\begin{array}{l}\text { Dingemans et al., } \\
2019\end{array}$ & $25(22)$ & $56(49)$ & 35.5 & 28.13 & BDI-II & $\begin{array}{l}\text { None-to-mild depressive symptoms in BED group. } \\
\text { Depressive symptoms were correlated with overall } \\
\text { neuropsychological inefficiencies }\end{array}$ & $\begin{array}{l}\text { BED group: psychiatric disorder not } \\
\text { specified }(\mathrm{n}=10)\end{array}$ & $\begin{array}{l}\text { Cognitive Flexibility: WCST (perseverative errors), } \\
\text { TMT (B-A). } \\
\text { Working Memory: BDS, FDS } \\
\text { Inhibition: BRIEF-A (behavioral index), Stroop test } \\
\text { (trial3/trial1) } \\
\text { Others: RCFT (central coherence), IGT (total net } \\
\text { score) }\end{array}$ \\
\hline $\begin{array}{l}\text { Duchesne et al., } \\
2010\end{array}$ & $38(29)$ & $38(34)$ & 34.35 & 36.24 & - & - & $\begin{array}{l}\text { Whole sample: specific phobia (12\%), } \\
\text { generalized anxiety disorder ( } 10 \%) \text {, } \\
\text { dysthymic disorder (6\%), panic } \\
\text { disorder ( } 9 \%) \text {, agoraphobia ( } 4 \% \text { ), } \\
\text { social phobia (5\%), alcohol abuse/ } \\
\text { dependence (3\%), posttraumatic stress } \\
\text { disorder ( } 3 \%) \text {, premenstrual } \\
\text { dysphoric disorder (2\%), } \\
\text { obsessive-compulsive disorder ( } 2 \%) \text {, } \\
\text { and others (1\%) }\end{array}$ & $\begin{array}{l}\text { Cognitive Flexibility: WCST (perseverative errors), } \\
\text { TMT-B, BADS Rule Shift Cards ( }{ }^{\circ} \text { errors) } \\
\text { Working Memory: BDS, FDS } \\
\text { Inhibition: Stroop test (correct answers, color-word } \\
\text { trial) } \\
\text { Others: BADS Action Program Test ( } \mathrm{n}^{\circ} \text { stages } \\
\text { completed), BADS Zoo Map Test (trial } 1, \mathrm{n}^{\circ} \text { errors), } \\
\text { BADS Modified Six Elements }\end{array}$ \\
\hline $\begin{array}{l}\text { Eneva et al., } \\
2017\end{array}$ & $55(55)$ & 77(77) & 32.1 & 28.4 & - & $\begin{array}{l}\text { Presence of psychiatric disorders did not } \\
\text { significantly correlate with performance on any } \\
\text { executive function measures }\end{array}$ & $\begin{array}{l}\text { BED group: mood disorder }(\mathrm{n}=5) \text {, } \\
\text { anxiety }(\mathrm{n}=10)\end{array}$ & $\begin{array}{l}\text { Cognitive Flexibility: DKEFS-trail making switching, } \\
\text { NIH dimensional change card sort } \\
\text { Working Memory: NIH working memory } \\
\text { Inhibition: DKEFS color word interference } \\
\text { inhibition, NIH flanker inhibitory control } \\
\text { Others: DKEFS verbal fluency, design fluency and } \\
\text { tower achievement }\end{array}$ \\
\hline $\begin{array}{l}\text { Grant \& } \\
\text { Chamberlain, } \\
2020\end{array}$ & $17(11)$ & $17(11)$ & 24.62 & 32.63 & $\begin{array}{l}\text { HAM-D, } \\
\text { HAM-A }\end{array}$ & $\begin{array}{l}\text { BED group reported subclinical levels of depression } \\
\text { and anxiety. No further control }\end{array}$ & $\begin{array}{l}\text { BED group: agoraphobia }(\mathrm{n}=2) \\
\text { social phobia }(\mathrm{n}=1) \text {, cannabis use } \\
\text { disorder }(\mathrm{n}=1) \text {, antisocial personality } \\
\text { disorder }(\mathrm{n}=1)\end{array}$ & $\begin{array}{l}\text { Cognitive Flexibility: IED (total errors, adjusted for } \\
\text { stages not completed) } \\
\text { Working Memory: One Touch Stockings of } \\
\text { Cambridge task (problems solved on first choice), } \\
\text { Spatial Working Memory Task* (total errors) } \\
\text { Inhibition: SST (stop-signal reaction time) } \\
\text { Others: CGT (quality of decision making) }\end{array}$ \\
\hline Kittel et al., 2017 & $22(18)$ & $44(36)$ & 14.99 & $85.66^{*}$ & BDI-II & $\begin{array}{l}\text { S-Diff in depression symptoms between groups. } \\
\text { Affective symptoms were not correlated with } \\
\text { neuropsychological test scores }\end{array}$ & - & $\begin{array}{l}\text { Cognitive Flexibility: СTMT (complex sequencing, } \mathrm{T} \\
\text { values) } \\
\text { Inhibition: Color-word interference } \\
\text { Others: IGT (total net score) }\end{array}$ \\
\hline
\end{tabular}

S-Diff in depression between groups. Results tested before and after cova

S-Diff in depression symptoms between groups. No

further control as covariate had no effect on IGT Depressive symptoms were correlated with overall neuropsychological inefficiencies ( and others $(1 \%)$

disorder $(\mathrm{n}=5)$ significantly correlate with performance on any

BED group: agoraphobia ( $\mathrm{n}=2$ ) isorder $(\mathrm{n}=1)$, antisocial personality isorder $(\mathrm{n}=1)$ 


\begin{tabular}{|c|c|c|c|c|c|c|c|c|}
\hline Study & $\begin{array}{l}\text { BED n } \\
\text { (females) }\end{array}$ & $\begin{array}{l}\text { Controls n } \\
\text { (females) }\end{array}$ & Pooled age & $\begin{array}{l}\text { Pooled } \\
\text { BMI }\end{array}$ & \multicolumn{2}{|c|}{ Evaluation of psychological comorbidities } & Current psychiatric comorbidities & Outcomes $\bullet$ \\
\hline $\begin{array}{l}\text { Kollei et al., } \\
2018\end{array}$ & $48(37)$ & $96(65)$ & 38.76 & 36.32 & BDI-II & $\begin{array}{l}\text { S-Diff in depression between groups. Depressive } \\
\text { symptoms as covariate. Multivariate effect of } \\
\text { depression was non-significant }\end{array}$ & $\begin{array}{l}\text { Whole sample: major depressive } \\
\text { disorder }(\mathrm{n}=17) \text {, anxiety disorder ( } \mathrm{n} \\
=24) \text {, posttraumatic stress disorder }(\mathrm{n} \\
=4) \text { obsessive-compulsive disorder } \\
(\mathrm{n}=2)\end{array}$ & $\begin{array}{l}\text { Cognitive Flexibility: IED (total errors) } \\
\text { Others: CGT (quality of decision making) }\end{array}$ \\
\hline Lee et al., 2017 & $13(13)$ & 14(14) & 23.44 & 22.9 & BDI-II & $\begin{array}{l}\text { S-Diff in depression and anxiety symptoms between } \\
\text { groups. No further control }\end{array}$ & - & $\begin{array}{l}\text { Inhibition: Stroop match-to-sample task (accuracy, } \\
\text { neutral stimuli) }\end{array}$ \\
\hline $\begin{array}{l}\text { Müller et al., } \\
2014\end{array}$ & $34(26)$ & $34(26)$ & 38.35 & 46.65 & PHQ-9 & $\begin{array}{l}\text { S-Diff in depression between groups. Results tested } \\
\text { before and after covarying for depression (no } \\
\text { differences found) }\end{array}$ & - & $\begin{array}{l}\text { Working Memory: Corsi Block Tapping Test (correct } \\
\text { answers) } \\
\text { Others: IGT (total net score) }\end{array}$ \\
\hline Oliva et al., 2019 & $21(17)$ & $21(16)$ & 24.56 & 21.79 & - & - & - & $\begin{array}{l}\text { Inhibition: GNG (reaction time, neutral condition), } \\
\text { SST (stop-signal reaction time, neutral condition) }\end{array}$ \\
\hline $\begin{array}{l}\text { Preuss et al., } \\
2019\end{array}$ & $24(21)$ & $77(66)$ & 37.39 & 30.34 & $\begin{array}{l}\text { PHQ-9, } \\
\text { BDI-II }\end{array}$ & $\begin{array}{l}\text { S-Diff in depression between groups. No further } \\
\text { control }\end{array}$ & $\begin{array}{l}\text { Whole sample: depressive disorder ( } \mathrm{n} \\
=16) \text {, anxiety disorder }(\mathrm{n}=15) \text {, } \\
\text { borderline personality disorder }(\mathrm{n}= \\
\text { 5), attention deficit hyperactivity } \\
\text { disorder }(\mathrm{n}=3 \text { ), skin picking disorder } \\
(\mathrm{n}=1) \text {, substance abuse }(\mathrm{n}=1)\end{array}$ & $\begin{array}{l}\text { Inhibition: SST (stop-signal reaction time) } \\
\text { Others: DOT (opened doors) }\end{array}$ \\
\hline $\begin{array}{l}\text { Reiter et al., } \\
2017\end{array}$ & $22(16)$ & $22(15)$ & 28.4 & 27.16 & - & - & - & $\begin{array}{l}\text { Cognitive Flexibility: TMT-B } \\
\text { Working Memory: BDS } \\
\text { Others: Digit- Symbol-Substitution Test, Matrices } \\
\text { Test }\end{array}$ \\
\hline $\begin{array}{l}\text { Rouel et al., } \\
2016\end{array}$ & $70(58)$ & $28(22)$ & 41.35 & 39.3 & DASS-21 & $\begin{array}{l}\text { NS-Diff in depression. anxiety and stress symptoms } \\
\text { between groups }\end{array}$ & - & $\begin{array}{l}\text { Cognitive Flexibility: WCST (perseverative errors), } \\
\text { TMT (B-A) } \\
\text { Working Memory: BDS } \\
\text { Others: RCFT }\end{array}$ \\
\hline $\begin{array}{l}\text { Steward et al., } \\
2017\end{array}$ & $24(24)$ & $80(80)$ & 25.45 & 25.6 & - & - & - & $\begin{array}{l}\text { Others: Monetary choice task (delay discounting: } \mathrm{k} \\
\text { overall values) }\end{array}$ \\
\hline $\begin{array}{l}\text { Svaldi et al., } \\
2010\end{array}$ & $17(17)$ & $18(18)$ & 40.3 & 31.72 & BDI-II & $\begin{array}{l}\text { S-Diff in depression symptoms between groups. No } \\
\text { further control }\end{array}$ & $\begin{array}{l}\text { BED group: } 23.5 \% \text { major depression, } \\
17.6 \% \text { panic disorder with } \\
\text { agoraphobia, } 11.8 \% \text { social phobia, } \\
5.9 \% \text { post-traumatic stress disorder, } \\
5.9 \% \text { generalized anxiety disorder, } \\
5.9 \% \text { somatization disorder }\end{array}$ & $\begin{array}{l}\text { Cognitive Flexibility: TMT-proportional score(B-A/ } \\
\text { A) }\end{array}$ \\
\hline $\begin{array}{l}\text { Svaldi, Schmitz, } \\
\text { et al., } 2014\end{array}$ & $31(31)$ & $36(36)$ & 43.32 & 34.15 & BDI-II & $\begin{array}{l}\text { S-Diff in depression symptoms between groups. } \\
\text { Depression was not correlated with recent-probes } \\
\text { task }\end{array}$ & $\begin{array}{l}\text { BED group: affective and anxiety } \\
\text { disorders were the most prevalent } \\
\text { comorbidities ( } n \text { not specified) }\end{array}$ & Others: Recent-probes task (accuracy) \\
\hline $\begin{array}{l}\text { Svaldi, } \\
\text { Naumann, } \\
\text { et al., } 2014\end{array}$ & $31(31)$ & 29(29) & 42.88 & 34.03 & BDI-II & $\begin{array}{l}\text { S-Diff in depression between groups. Depression } \\
\text { was correlated with response time in all go trials }\end{array}$ & $\begin{array}{l}\text { Whole sample: major depression }(\mathrm{n}= \\
\text { 2), dysthymia }(\mathrm{n}=1) \text {, panic disorder } \\
(\mathrm{n}=2) \text {, social phobia }(\mathrm{n}=2) \text {, specific } \\
\text { phobia }(\mathrm{n}=2) \text {, obsessive compulsive } \\
\text { disorder }(\mathrm{n}=1) \text {, post-traumatic stress } \\
\text { disorder }(\mathrm{n}=1) \text {, pain disorder }(\mathrm{n}=1)\end{array}$ & $\begin{array}{l}\text { Inhibition: SST (neutral condition, commission } \\
\text { errors) }\end{array}$ \\
\hline Wu et al., 2013 & $54(49)$ & $74(72)$ & 36.63 & 31.46 & BDI-II & $\begin{array}{l}\text { S-Diff in depression symptoms between groups. } \\
\text { Results tested with and without BED patients on } \\
\text { psychotropic medication (no differences found). } \\
\text { Depression was not correlated with SSRT }\end{array}$ & $\begin{array}{l}\text { BED group: psychotropic medication } \\
(\mathrm{n}=19)\end{array}$ & $\begin{array}{l}\text { Inhibition: SST (stop-signal reaction time) } \\
\text { Others: GDT (total net score) }\end{array}$ \\
\hline
\end{tabular}

*BMI Percentile, **BMI z-score.

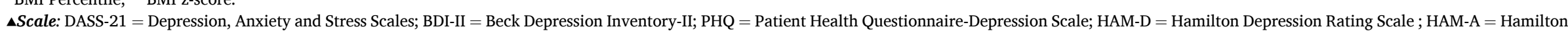
Anxiety Rating Scale ; IDS-SR = Inventory for Depressive Symptomatology Self-Rated.

Reported affective symptoms: S-Diff $=$ Significant differences; NS-Diff $=$ Nonsignificant differences.

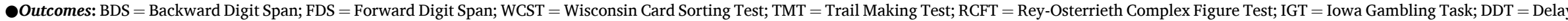

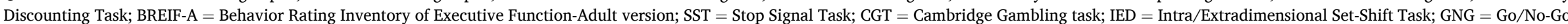
task; HSCT $=$ Hayling Sentence Completion Test; GDT = Game of Dice Task; DOT = Door Opening Task. 


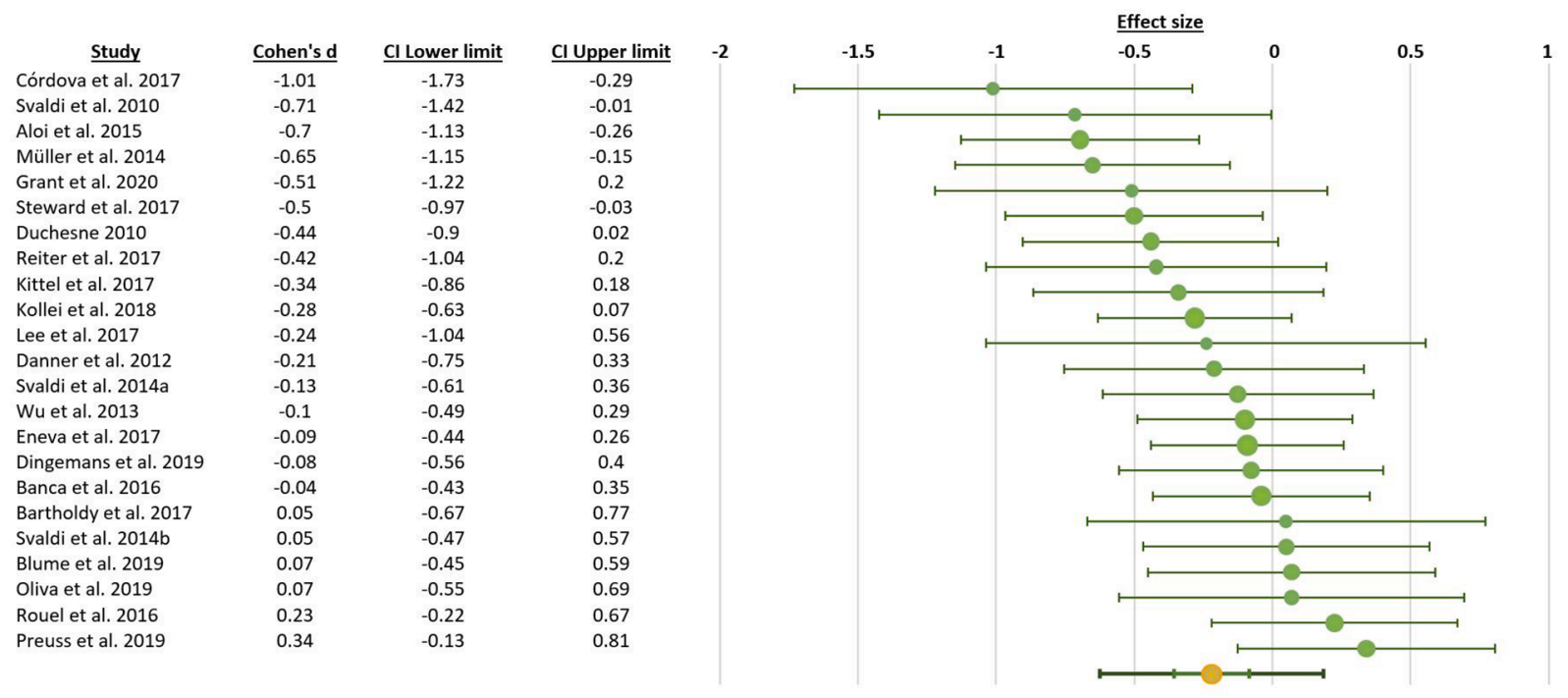

Fig. 2. Effect size (Cohen's d) and 95\% confidence interval for the relationship between BED and executive functions.

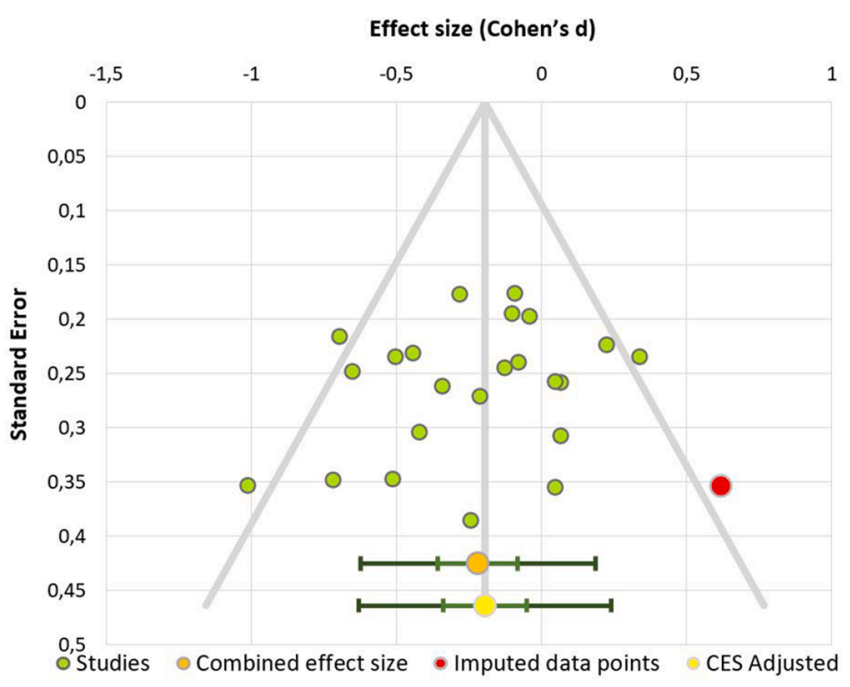

Fig. 3. Publication bias funnel plot of the first meta-analysis. Dots represent each study included.

introduced into the meta-analysis as two separate studies. As such, the final number of independent samples entered in the meta-analysis was 10. The statistical power of this meta-analysis was 27\% (Quintana, 2017). The meta-analysis results did not point to a significant association between uncontrolled eating and executive functions (mean correlation value $\mathrm{r}=-0.09$, confidence intervals: $-0.21,0.03 ; \mathrm{P}=0.082$ ) (Fig. 4). The heterogeneity value in the investigated studies was $\mathrm{I}^{2}=55.98 \%$, suggesting a moderate heterogeneity across studies. A funnel plot and Egger test did not provide evidence for publication bias $(\mathrm{t}=-1.14, \mathrm{P}=$ 0.29). Four missing studies were detected using the trim-and-fill method (Fig. 5). The inputs of the two meta-analyses are available here: htt ps://osf.io/uznve/.

\section{Discussion}

In the present study, we explored the relationship between binge eating behaviors and executive functions. Accordingly, we performed two main meta-analyses to analyze $i$ ) group differences between patients with BED and healthy controls in executive functions and ii) the relationship between binge eating patterns in non-clinical populations and executive functions. In line with our first hypothesis, patients with BED showed lower scores on executive functions than control subjects. The combined effect size obtained in the first meta-analysis was small ( $\mathrm{d}=$ -0.22). Neither age nor BMI acted as moderators in the results. Two additional meta-analyses focusing on inhibition and cognitive flexibility in this population were performed, not obtaining statistically significant differences between BED and healthy controls. Contrary to our second hypothesis, uncontrolled eating was not associated with executive functions when examined in non-clinical populations $(r=-0.09)$. This suggests that alterations in executive functions might strongly depend on the severity of the binge eating phenotype. That is, individuals with a clinical diagnosis of BED seem to show subtle alterations in general executive functions. At the same time, executive functions do not differ along the spectrum of non-clinical binge eating (i.e., uncontrolled eating) patterns.

Our results suggest that patients with a clinical diagnosis of BED show small differences in general executive performance, rather than in specific subdomains such as inhibition or cognitive flexibility. Nevertheless, the small number of studies available makes it difficult to reliably test whether some specific subdomain might be driving these results. A previous meta-analysis (Cury et al., 2020) suggested that BED patients showed worse performance on working memory tasks relative to healthy participants. Unfortunately, there were very few studies available to evaluate the subdomain of working memory in an appropriate manner.

Patients with BED face psychological comorbidities, including depression, anxiety, stress and impulse control difficulties (Udo \& Grilo,

Table 2

Additional meta-analysis results

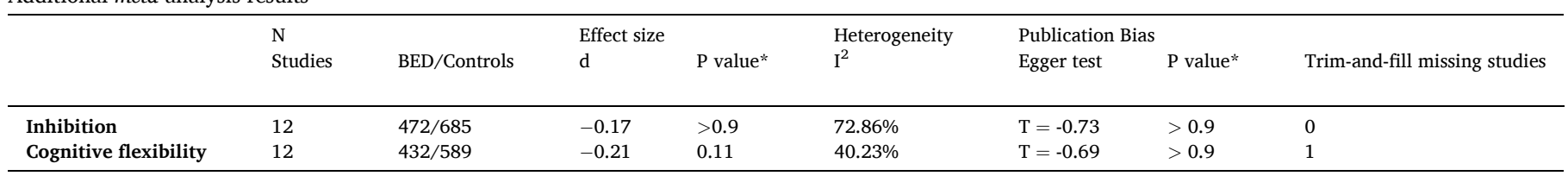

*Bonferroni corrected. 
Table 3

Description of studies included in the second meta-analysis.

\begin{tabular}{|c|c|c|c|c|c|c|c|c|c|}
\hline Study & $\begin{array}{l}\text { Experimental } \\
\text { group } \\
\mathrm{n} \text { (females) }\end{array}$ & $\begin{array}{l}\text { Control } \\
\text { group } \\
\mathrm{n} \\
\text { (females) }\end{array}$ & $\begin{array}{l}\text { Pooled } \\
\text { age }\end{array}$ & $\begin{array}{l}\text { Pooled } \\
\text { BMI }\end{array}$ & $\begin{array}{l}\text { Evaluat } \\
\text { Scale» }\end{array}$ & $\begin{array}{l}\text { n of psychological comorb } \\
\text { Reported affective } \\
\text { symptoms }\end{array}$ & $\begin{array}{l}\text { lities } \\
\text { Current } \\
\text { psychiatric } \\
\text { comorbidities }\end{array}$ & Eating scale $\times$ & Outcomes \\
\hline $\begin{array}{l}\text { Calvo et al., } \\
2014\end{array}$ & $30(18)$ & $32(17)$ & 21.13 & 28.77 & - & - & - & $\begin{array}{l}\text { TFEQ-R18 } \\
\text { (uncontrolled } \\
\text { eating) }\end{array}$ & $\begin{array}{l}\text { Inhibition: ANAM4 Go/ } \\
\text { No-Go task*(reaction } \\
\text { time) } \\
\text { Working Memory: } \\
\text { ANAM4 Running } \\
\text { Memory Continuous } \\
\text { Performance Task } \\
\text { (reaction time) }\end{array}$ \\
\hline $\begin{array}{l}\text { Goldschmidt } \\
\text { et al., } 2018\end{array}$ & $26(16)$ & $49(28)$ & 10.53 & $1.60^{* * *}$ & - & - & - & $\begin{array}{l}\text { Child EDE (LOC } \\
\text { eating frequency) }\end{array}$ & $\begin{array}{l}\text { Inhibition: BRIEF (T } \\
\text { scores) Dimensional } \\
\text { Change Card Sort } \\
\text { (accuracy and RT), } \\
\text { Flanker test (accuracy } \\
\text { and RT) } \\
\text { Working Memory: List } \\
\text { Sorting (accuracy) } \\
\text { Others: IGT (T score), } \\
\text { TOL (total moves) }\end{array}$ \\
\hline $\begin{array}{l}\text { Kelly et al., } \\
2013\end{array}$ & $50(50)$ & $66(66)$ & 19.15 & 23.86 & $\begin{array}{l}\text { CES- } \\
\text { D, } \\
\text { SAI }\end{array}$ & $\begin{array}{l}\text { NS-Diff in depression } \\
\text { and anxiety between } \\
\text { groups. Results tested } \\
\text { before and after } \\
\text { covarying for } \\
\text { depression and anxiety } \\
\text { (no differences found) }\end{array}$ & - & $\begin{array}{l}\text { EDE-Q (total } \\
\text { number of binge } \\
\text { episodes) }\end{array}$ & $\begin{array}{l}\text { Inhibition: CPT-II } \\
\text { (commission errors) }\end{array}$ \\
\hline $\begin{array}{l}\text { Maayan et al., } \\
2011\end{array}$ & $54(34)$ & $37(21)$ & 17.43 & 32.46 & - & - & - & $\begin{array}{l}\text { TFEQ } \\
\text { (disinhibition } \\
\text { factor) }\end{array}$ & $\begin{array}{l}\text { Inhibition: Stroop color- } \\
\text { word score }\end{array}$ \\
\hline $\begin{array}{l}\text { Macchi et al., } \\
2017\end{array}$ & $311(179)$ & - & 15.7 & $65.58^{*}$ & - & - & - & $\begin{array}{l}\text { DEBQ (emotional } \\
\text { eating, external } \\
\text { eating), QEWP-A, } \\
\text { PFS }\end{array}$ & Others: IGT (net score) \\
\hline $\begin{array}{l}\text { Rodrigue et al., } \\
2018\end{array}$ & $37(29)$ & $33(25)$ & 40.26 & 47.10 & $\begin{array}{l}\text { BDI-II, } \\
\text { STAI }\end{array}$ & $\begin{array}{l}\text { S-Diff in depression and } \\
\text { anxiety between } \\
\text { groups. Depression and } \\
\text { anxiety were correlated } \\
\text { with BRIEF scores }\end{array}$ & - & YFAS, BES & $\begin{array}{l}\text { Inhibition: BRIEF } \\
\text { Behavioral regulation } \\
\text { Others: BREIF } \\
\text { Metacognition, D-KEFS } \\
\text { Tower Test (moves } \\
\text { accuracy ratio) }\end{array}$ \\
\hline $\begin{array}{l}\text { Sims et al., } \\
2014\end{array}$ & $47(27)$ & - & 45.7 & 43.3 & BDI-II & $\begin{array}{l}\text { Depression within the } \\
\text { sample was relatively } \\
\text { low. Depression as } \\
\text { covariate }\end{array}$ & - & $\begin{array}{l}\text { EBPQ (emotional } \\
\text { eating) }\end{array}$ & $\begin{array}{l}\text { Inhibition: Stroop Test } \\
\text { (interference score), } \\
\text { WCST (perseverative } \\
\text { errors) }\end{array}$ \\
\hline $\begin{array}{l}\text { Steward et al., } \\
2018 \\
\text { (obesity) }\end{array}$ & $33(33)$ & - & 38.7 & 42.4 & - & - & - & YFAS & $\begin{array}{l}\text { Inhibition: CPT-II } \\
\text { (commission errors) } \\
\text { Others: IGT (total score) }\end{array}$ \\
\hline $\begin{array}{l}\text { Steward et al., } \\
2018 \\
\text { (normal- } \\
\text { weight) }\end{array}$ & $36(36)$ & - & 30.6 & 21.3 & - & - & - & YFAS & $\begin{array}{l}\text { Inhibition: CPT-II* } \\
\text { (commission errors) } \\
\text { Others: IGT (total score) }\end{array}$ \\
\hline $\begin{array}{l}\text { Watson \& } \\
\text { Garvey, } 2013\end{array}$ & $48(32)$ & - & 21.63 & 23.38 & - & - & - & $\begin{array}{l}\text { DEBQ (emotional } \\
\text { eating, external } \\
\text { eating) }\end{array}$ & $\begin{array}{l}\text { Inhibition: Stroop Test } \\
\text { (interference score) }\end{array}$ \\
\hline
\end{tabular}

*BMI Percentile, **BMI z-score.

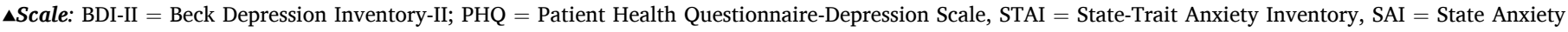
Inventory, CES-D = Center for Epidemiological Studies Depression Scale.

$\square$ Reported affective symptoms: S-Diff = Significant differences; NS-Diff = Nonsignificant differences.

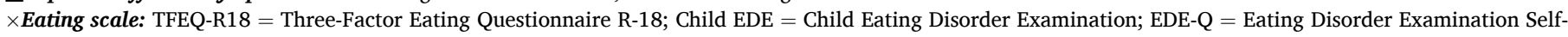

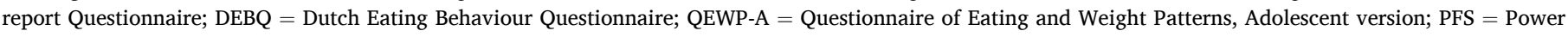
of Food Scale; YFAS = Yale Food Addiction Scale; BES = Binge Eating Scale; EBPQ = Eating Behavior Patterns Questionnaire.

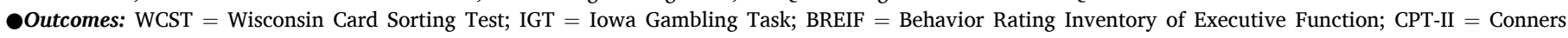
Continuous Performance Test; TOL = Tower of London.

2019). There is a consistent association between BED and emotional distress in the literature (Dingemans et al., 2017; Nicholls et al., 2016). Mood disorders, such as depression and anxiety, have been linked with impairments in executive functions. For example, (Ajilchi \& Nejati, 2017) compared executive functions in healthy volunteers and individuals with depression, anxiety and stress. They reported that, relative to the control group, the three clinical groups showed worse performance in memory, inhibition, planning, and flexibility, without differences between them. Cognitive deficits in mood disorders, moreover, might persist in remission and even worsen with repeated episodes, as it has been shown in major depressive disorder (Semkovska et al., 2019). These close links between binge eating and mood disorders make it almost impossible to disentangle whether cognitive differences are due to BED or to the emotional distress that this diagnostic entails. 


\begin{tabular}{|c|c|c|c|}
\hline Study & Correlation & CI Lower limit & Cl Upper limit \\
\hline Steward et al. 2018 (obesity) & -0.30 & -0.52 & -0.05 \\
\hline Steward et al. 2018 (normal-weight) & -0.30 & -0.48 & -0.10 \\
\hline Calvo et al. 2014 & -0.25 & -0.51 & 0.05 \\
\hline Goldschimidt et al. 2018 & -0.18 & -0.50 & 0.19 \\
\hline Sims et al.2014 & -0.12 & -0.35 & 0.12 \\
\hline Rodrigue et al. 2018 & -0.06 & -0.39 & 0.29 \\
\hline Macchi et al. 2017 & -0.06 & -0.34 & 0.24 \\
\hline Maayan et al. 2011 & -0.01 & -0.12 & 0.10 \\
\hline Watson et al. 2013 & 0.00 & -0.23 & 0.23 \\
\hline Kelly et al. 2013 & 0.20 & 0.02 & 0.37 \\
\hline
\end{tabular}

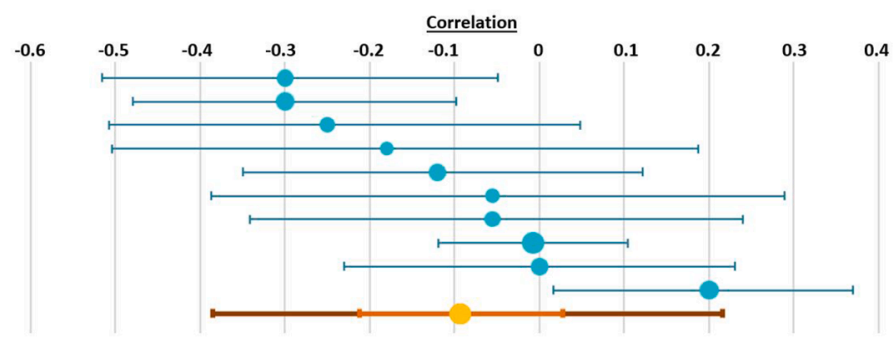

Fig. 4. Correlation coefficients and $95 \%$ confidence intervals for the relationship between uncontrolled eating and executive functions.

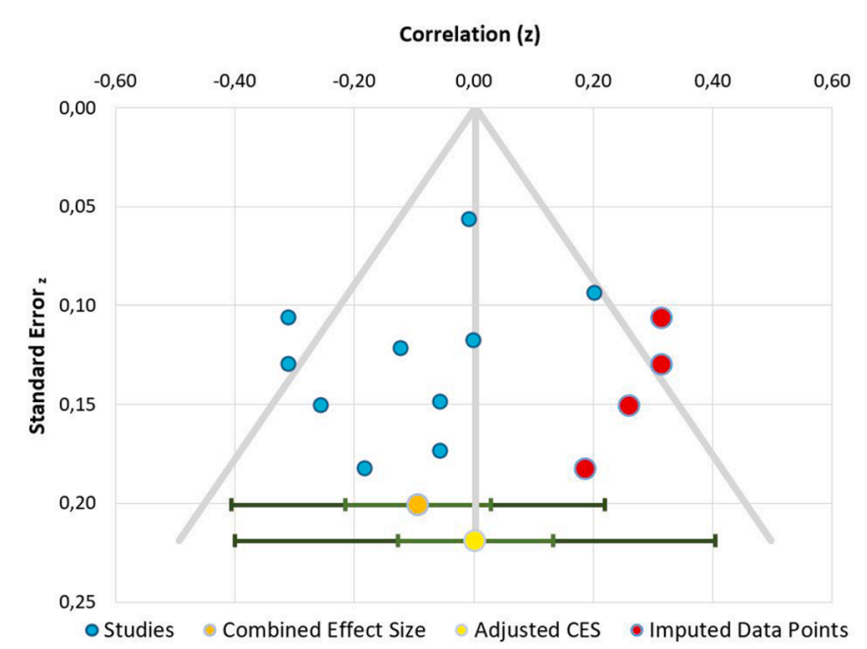

Fig. 5. Publication bias funnel plot of the second meta-analysis. Note that although in there are 10 dots, only 9 studies were included. That is because Steward et al. provided separated results for normal-weight and obese participants.

The relationship between BED and lower performance in executive functions might have clinical implications. Intervention studies have applied cognitive control training in order to ameliorate pathological eating behaviors (Yang et al., 2019). For instance, some studies have shown reductions in binge eating frequency after the completion of inhibitory control and strategy planning training programs (Chami et al., 2020; Yang et al., 2019). This suggests that cognitive interventions based on executive function training might be a promising treatment to BED.

While performing this study, we have identified a number of points that remain unanswered and that we hope that they will be addressed in the near future. First, future research should examine the different subdomains of executive function to have a more holistic view of this neurocognitive domain in BED. This is specially so in the case of working memory. As aforementioned, very few studies have evaluated differences in working memory with standardized measurements so far. Second, studies should examine executive functions in BED patients with varying degrees of affective comorbidity. This could help us to obtain a more precise cognitive profile of patients with BED and with affective symptomatology. Third, the cross-sectional nature of this study does not allow us to know if the link between binge eating behaviors and executive functions show different trajectories over time. For instance, it would be interesting to know if participants with low executive functions might have higher probabilities of suffering from BED. Therefore, future studies with longitudinal designs as well as clinical studies are needed in order to determine the clinical and prognostic value of executive functions in individuals with BED.

Some limitations of the present study must be highlighted. First, we have included some papers reporting psychiatric comorbidities in the meta-analysis. Also, few articles included used both behavioral tasks and questionnaires to evaluate executive functions. Second, a small number of studies were included in the second meta-analysis, and as such, its statistical power was limited. Its results should then be considered preliminary. Third, we included both bivariate and partial correlations in the second meta-analysis. This might be a source of heterogeneity across studies, since the original linear models were built with different covariates, and they represent a different population parameter (Aloe \& Thompson, 2013). Fourth, the validity of a meta-analysis depends on the quality of the included studies. However, we did not perform a systematic quality assessment of the studies as part of the screening process. In this regard, we refer the interested reader to Lavagnino et al., 2016, where the authors reviewed and scored studies on inhibitory control in obesity and BED according to a modified version of the NewcastleOttawa Scale for quality assessment.

\section{Conclusions}

The study of neurocognitive characteristics associated with binge eating behaviors might guide clinical strategies to prevent and treat BED - the most prevalent eating-related psychopathology. The present study has focused on executive functions as a possible correlate of binge eating and has tested two hypotheses. First, whether patients with BED show deficits in executive function performance, relative to control participants. Second, we hypothesized that deficits would be observed at a nonclinical level. We examined the correlation between uncontrolled eating, a psychobiological construct considered to reflect binge eating behaviors, and executive functions in general populations. According to our first hypothesis, patients with BED performed worse than controls in general executive functions. The magnitude of this difference was small. Contrary to our second hypothesis, uncontrolled eating patterns in nonclinical populations were not associated with executive functions. As such, small deficits in executive functions might be associated with severe phenotypes of binge eating. We speculate that the close links between BED and emotional distress might partly explain these cognitive deficits.

\section{Data availability}

Link to the preregistration of the study: https://osf.io/trn4b. Link to the input of the meta-analysis: https://osf.io/uznvc/.

\section{Funding sources}

María Ángeles Jurado received funding from the Spanish Ministry of Economy, Industry and Competitiveness (PSI2017-86536-C2-1-R). Anna Prunell i Castañé received a PhD scholarship from the Spanish Ministry of Science, Innovation and Universities (PRE2019-087430).

\section{CRediT authorship contribution statement}

Anna Prunell-Castañé: Conceptualization, Formal analysis, Investigation, Writing - original draft, Visualization. María Ángeles Jurado: Conceptualization, Resources, Writing - review \& editing, Supervision, 
Funding acquisition. Isabel García-García: Conceptualization, Investigation, Data curation, Writing - original draft, Supervision, Project administration.

\section{Declaration of Competing Interest}

The authors declared that there is no conflict of interest.

\section{Appendix A. Supplementary data}

Supplementary data to this article can be found online at https://doi. org/10.1016/j.abrep.2020.100337.

\section{References}

Ajilchi, B., \& Nejati, V. (2017). Executive functions in students with depression, anxiety, and stress symptoms. Basic and Clinical Neuroscience, 8(3), 223-232. https://doi.org/ 10.18869/nirp.bcn.8.3.223.

Aloe, A. M., \& Thompson, C. G. (2013). The Synthesis of Partial Effect Sizes. Journal of the Society for Social Work and Research, 4(4), 390-405. https://doi.org/10.5243/ jsswr.2013.24.

Aloi, M., Rania, M., Caroleo, M., Bruni, A., Palmieri, A., Cauteruccio, M. A., De Fazio, P., \& Segura-García, C. (2015). Decision making, central coherence and set-shifting: A comparison between Binge Eating Disorder, Anorexia Nervosa and Healthy Controls. BMC Psychiatry, 15(1), 1-10. https://doi.org/10.1186/s12888-015-0395-z.

Banca, P., Harrison, N. A., \& Voon, V. (2016). Compulsivity Across the Pathological Misuse of Drug and Non-Drug Rewards. Frontiers in Behavioral Neuroscience, 10(154). https://doi.org/10.3389/fnbeh.2016.00154.

Bartholdy, S., Rennalls, S., Danby, H., Jacques, C., Campbell, I. C., Schmidt, U., \& O'Daly, O. G. (2017). Temporal Discounting and the Tendency to Delay Gratification across the Eating Disorder Spectrum. European Eating Disorders Review, 25(5), 344-350. https://doi.org/10.1002/erv.2513.

Blume, M., Schmidt, R., \& Hilbert, A. (2018). Executive Functioning in Obesity, Food Addiction, and Binge-Eating Disorder. Nutrients, 11(1), 54. https://doi.org/10.3390/ nu11010054.

Calvo, D., Galioto, R., Gunstad, J., \& Spitznagel, M. B. (2014). Uncontrolled eating is associated with reduced executive functioning. Clinical Obesity, 4(3), 172-179. https://doi.org/10.1111/cob.12058.

Chami, R., Cardi, V., Lawrence, N., Macdonald, P., Rowlands, K., Hodsoll, J., \& Treasure, J. (2020). Targeting binge eating in bulimia nervosa and binge eating disorder using inhibitory control training and implementation intentions: A feasibility trial. Psychological Medicine. https://doi.org/10.1017/ S0033291720002494.

Córdova, M. E., Schiavon, C. C., Busnello, F. M., \& Reppold, C. T. (2017). Nutritional and Neuropsychological Profile of the Executive Functions on Binge Eating Disorder in Obese Adults. Nutrición Hospitalaria, 34(6), 1448-1454. https://doi.org/10.20960/ nh.1151.

Cury, M. E. G., Berberian, A., Sini Scarpato, B., Kerr-Gaffney, J., Santos, F. H., \& Medeiros Claudino, A. (2020). Scrutinizing domains of executive function in binge eating disorder: A systematic review and meta-analysis. Frontiers in Psychiatry, 11(288), 1-15. https://doi.org/10.3389/fpsyt.2020.00288.

Danner, U. N., Ouwehand, C., Van Haastert, N. L., Hornsveld, H., \& De Ridder, D. T. D. (2012). Decision-making impairments in women with binge eating disorder in comparison with obese and normal weight women. European Eating Disorders Review, 20(1). https://doi.org/10.1002/erv.1098.

de Zwaan, M. (2001). Binge eating disorder and obesity. International Journal of Obesity, 25, S51-S55. https://doi.org/10.1038/sj.ijo.0801699.

Diagnostic and Statistical Manual of Mental Disorders (Fifth Edit). (2013). American Psychiatric Association.

Diamond, A. (2013). Executive functions. Annual Review of Psychology, 64, 136-168. https://doi.org/10.1146/annurev-psych-113011-143750.

Dingemans, A., Danner, U., \& Parks, M. (2017). Emotion regulation in binge eating disorder: A review. Nutrients, 9(11), 1-11. https://doi.org/10.3390/nu9111274.

Dingemans, A. E., Vanhaelen, C. B., Aardoom, J. J., \& van Furth, E. F. (2019). The influence of depressive symptoms on executive functioning in binge eating disorder: A comparison of patients and non-obese healthy controls. Psychiatry Research, 274, 138-145. https://doi.org/10.1016/j.psychres.2019.02.033.

Duchesne, M., Mattos, P., Appolinário, J. C., et al. (2010). Assessment of executive functions in obese individuals with binge eating disorder. Brazilian Journal of Psychiatry, 32(4), 381-388.

Emery, R. L., \& Levine, M. D. (2017). Questionnaire and behavioral task measures of impulsivity are differentially associated with body mass index: A comprehensive meta-analysis. Psychological Bulletin, 143(8), 868-902. https://doi.org/10.1037/ bul0000105.

Eneva, K. T., Arlt, J. M., Yiu, A., Murray, S. M., \& Chen, E. Y. (2017). Assessment of executive functioning in binge-eating disorder independent of weight status. International Journal of Eating Disorders, 50(8), 942-951. https://doi.org/10.1002/ eat. 22738 .

García-García, I., Morys, F., Michaud, A., \& Dagher, A. (2020). Food addiction, skating on thin ice. A critical overview of neuroimaging findings. Current Addiction Reports, 7, 20-29.
Goldschmidt, A. B., O'Brien, S., Lavender, J. M., Pearson, C. M., Le Grange, D., \& Hunter, S. J. (2018). Executive functioning in a racially diverse sample of children who are overweight and at risk for eating disorders. Appetite, 124, 43-49. https:// doi.org/10.1016/j.appet.2017.03.010.

Grant, J. E., \& Chamberlain, S. R. (2020). Neurocognitive findings in young adults with binge eating disorder. International Journal of Psychiatry in Clinical Practice, 24(1), 71-76. https://doi.org/10.1080/13651501.2019.1687724.

Higgins, J. P. T., Thompson, S. G., Deeks, J. J., \& Altman, D. G. (2003). Measuring inconsistency in meta-analyses. British Medical Journal, 327, 557-560. https://doi. org/10.1136/bmj.327.7414.557.

Kakoschke, N., Aarts, E., \& Verdejo-García, A. (2019). The Cognitive Drivers of Compulsive Eating Behavior. Frontiers in Behavioral Neuroscience, 12, 1-8. https:// doi.org/10.3389/fnbeh.2018.00338.

Kelly, N. R., Bulik, C. M., \& Mazzeo, S. E. (2013). Executive functioning and behavioral impulsivity of young women who binge eat. International Journal of Eating Disorders, 46(2), 127-139. https://doi.org/10.1002/eat.22096.

Kessler, R. C., Berglund, P. A., Chiu, W. T., Deitz, A. C., Hudson, J. I., Shahly, V., .. Xavier, M. (2013). The prevalence and correlates of binge eating disorder in the World Health Organization World Mental Health Surveys. Biological Psychiatry, 73 (9), 904-914. https://doi.org/10.1016/j.biopsych.2012.11.020.

Kessler, R. M., Hutson, P. H., Herman, B. K., \& Potenza, M. N. (2016). The neurobiological basis of binge-eating disorder. Neuroscience \& Biobehavioral Reviews, 63, 223-238. https://doi.org/10.1016/j.neubiorev.2016.01.013.

Kittel, R., Schmidt, R., \& Hilbert, A. (2017). Executive functions in adolescents with binge-eating disorder and obesity. International Journal of Eating Disorders, 50(8), 933-941. https://doi.org/10.1002/eat.22714.

Kollei, I., Rustemeier, M., Schroeder, S., Jongen, S., Herpertz, S., \& Loeber, S. (2018). Cognitive control functions in individuals with obesity with and without bingeeating disorder. International Journal of Eating Disorders, 51(3), 233-240. https://doi. org/10.1002/eat.22824.

Kozak, M. J., \& Cuthbert, B. N. (2016). The NIMH Research Domain Criteria Initiative: Background, Issues, and Pragmatics. Psychophysiology, 53(3), 286-297. https://doi. org/10.1111/psyp.12518.

Lavagnino, L., Arnone, D., Cao, B., Soares, J. C., \& Selvaraj, S. (2016). Inhibitory control in obesity and binge eating disorder: A systematic review and meta-analysis of neurocognitive and neuroimaging studies. Neuroscience \& Biobehavioral Reviews, 68 , 714-726. https://doi.org/10.1016/j.neubiorev.2016.06.041.

Lee, J. E., Namkoong, K., \& Jung, Y. C. (2017). Impaired prefrontal cognitive control over interference by food images in binge-eating disorder and bulimia nervosa. Neuroscience Letters, 651, 95-101. https://doi.org/10.1016/j.neulet.2017.04.054.

Liberati, A., Altman, D. G., Tetzlaff, J., Mulrow, C., Gøtzsche, P. C., Ioannidis, J. P. A., Clarke, M., Devereaux, P. J., Kleijnen, J., \& Moher, D. (2009). The PRISMA statement for reporting systematic reviews and meta-analyses of studies that evaluate health care interventions: Explanation and elaboration. PLoS Medicine, 6(7). https://doi. org/10.1371/journal.pmed.1000100.

Maayan, L., Hoogendoorn, C., Sweat, V., \& Convit, A. (2011). Disinhibited eating in obese adolescents is associated with orbitofrontal volume reductions and executive dysfunction. Obesity, 19(7), 1382-1387. https://doi.org/10.1038/oby.2011.15.

Macchi, R., MacKew, L., \& Davis, C. (2017). Is decision-making ability related to food choice and facets of eating behaviour in adolescents? Appetite, 116, 442-455. https://doi.org/10.1016/j.appet.2017.05.031.

Manasse, S. M., Espel, H. M., Schumacher, L. M., Kerrigan, S. G., Zhang, F., Forman, E. M., \& Juarascio, A. S. (2016). Does impulsivity predict outcome in treatment for binge eating disorder? A multimodal investigation. Appetite, 105, 172-179. https://doi.org/10.1016/j.appet.2016.05.026.

Manasse, S. M., Goldstein, S. P., Wyckoff, E., Forman, E. M., Juarascio, A. S., Butryn, M. L., Ruocco, A. C., \& Nederkoorn, C. (2016). Slowing down and taking a second look: Inhibitory deficits associated with binge eating are not food-specific. Appetite, 96, 555-559. https://doi.org/10.1016/j.appet.2015.10.025.

Müller, A., Brandl, C., Kiunke, W., Georgiadou, E., Horbach, T., Köhler, H., \& De Zwaan, M. (2014). Food-independent tendency to disadvantageous decisions in obese individuals with regular binge eating. Comprehensive Psychiatry, 55(1), 64-70. https://doi.org/10.1016/j.comppsych.2013.08.010.

Nicholls, W., Devonport, T. J., \& Blake, M. (2016). The association between emotions and eating behaviour in an obese population with binge eating disorder. Obesity Reviews, 17(1), 30-42. https://doi.org/10.1111/obr.12329.

Oliva, R., Morys, F., Horstmann, A., Castiello, U., \& Begliomini, C. (2019). The impulsive brain: Neural underpinnings of binge eating behavior in normal-weight adults. Appetite, 136, 33-49. https://doi.org/10.1016/j.appet.2018.12.043.

Preuss, H., Leister, L., Pinnow, M., \& Legenbauer, T. (2019). Inhibitory control pathway to disinhibited eating: A matter of perspective? Appetite, 141. https://doi.org/ 10.1016/j.appet.2019.05.028.

Quintana, D. (2017). How to calculate statistical power for your meta-analysis. Towards Data Science. https://towardsdatascience.com/how-to-calculate-statistical-powerfor-your-meta-analysis-e108ee586ae8.

Reiter, A. M. F., Heinze, H. J., Schlagenhauf, F., \& Deserno, L. (2017). Impaired Flexible Reward-Based Decision-Making in Binge Eating Disorder: Evidence from Computational Modeling and Functional Neuroimaging. Neuropsychopharmacology, 42(3), 628-637. https://doi.org/10.1038/npp.2016.95.

Rodrigue, C., Ouellette, A. S., Lemieux, S., Tchernof, A., Biertho, L., \& Bégin, C. (2018). Executive functioning and psychological symptoms in food addiction: A study among individuals with severe obesity. Eating and Weight Disorders, 23(4), 469-478. https:// doi.org/10.1007/s40519-018-0530-1.

Rouel, M., Raman, J., Hay, P., \& Smith, E. (2016). Validation of the Behaviour Rating Inventory of Executive Function - Adult Version (BRIEF-A) in the obese with and 
without binge eating disorder. Eating Behaviors, 23, 58-65. https://doi.org/10.1016/ j.eatbeh.2016.07.010.

Sachdev, P. S., Blacker, D., Blazer, D. G., Ganguli, M., Jeste, D. V., Paulsen, J. S., \& Petersen, R. C. (2014). Classifying neurocognitive disorders: The DSM-5 approach. Nature Reviews Neurology, 10(11), 634-642. https://doi.org/10.1038/ nrneurol.2014.181.

Semkovska, M., Quinlivan, L., O'Grady, T., Johnson, R., Collins, A., O'Connor, J., Knittle, H., Ahern, E., \& Gload, T. (2019). Cognitive function following a major depressive episode: A systematic review and meta-analysis. The Lancet Psychiatry, 6 (10), 851-861. https://doi.org/10.1016/S2215-0366(19)30291-3.

Sims, R. C., Bennett, N.-K., Denee, ;, Mwendwa, T., Ali, M. K., Levy, S.-A. T., Callender, C. O., \& Campbell, A. L. (2014). Executive function and negative eating behaviors in severely obese African Americans. Ethn Dis, 24(3), 328-334.

Steward, T., Mestre-Bach, G., Vintró-Alcaraz, C., Agüera, Z., Jiménez-Murcia, S., Granero, R., \& Fernández-Aranda, F. (2017). Delay Discounting of Reward and Impulsivity in Eating Disorders: From Anorexia Nervosa to Binge Eating Disorder. European Eating Disorders Review, 25(6), 601-606. https://doi.org/10.1002/ erv.2543.

Steward, T., Mestre-Bach, G., Vintró-Alcaraz, C., Lozano-Madrid, M., Agüera, Z., Fernández-Formoso, J. A., Granero, R., Jiménez-Murcia, S., Vilarrasa, N., GarcíaRuiz-de-Gordejuela, A., Veciana de las Heras, M., Custal, N., Virgili, N., LópezUrdiales, R., Gearhardt, A. N., Menchón, J. M., Soriano-Mas, C., \& FernándezAranda, F. (2018). Food addiction and impaired executive functions in women with obesity. European Eating Disorders Review, 26(6), 574-584. https://doi.org/ 10.1002/erv.2636.

Suurmond, R., van Rhee, H., \& Hak, T. (2017). Introduction, comparison, and validation of Meta-Essentials: A free and simple tool for meta-analysis. In Research Synthesis Methods (Vol. 8, Issue 4, pp. 537-553). John Wiley \& Sons, Ltd. https://doi.org/ 10.1002/jrsm.1260.

Svaldi, J., Brand, M., \& Tuschen-Caffier, B. (2010). Decision-making impairments in women with binge eating disorder. Appetite, 54(1), 84-92. https://doi.org/10.1016/ j.appet.2009.09.010.

Svaldi, J., Naumann, E., Trentowska, M., \& Schmitz, F. (2014). General and food-specific inhibitory deficits in binge eating disorder. International Journal of Eating Disorders, 47(5), 534-542. https://doi.org/10.1002/eat.22260.
Svaldi, J., Schmitz, F., Trentowska, M., Tuschen-Caffier, B., Berking, M., \& Naumann, E. (2014). Cognitive interference and a food-related memory bias in binge eating disorder. Appetite, 72, 28-36. https://doi.org/10.1016/j.appet.2013.09.014.

Udo, T., \& Grilo, C. M. (2018). Prevalence and correlates of DSM-5-defined eating disorders in a nationally representative sample of U.S. adults. Biological Psychiatry, 84(5), 345-354. https://doi.org/10.1016/j.biopsych.2018.03.014.

Udo, T., \& Grilo, C. M. (2019). Psychiatric and medical correlates of DSM-5 eating disorders in a nationally representative sample of adults in the United States. International Journal of Eating Disorders, 52(1), 42-50. https://doi.org/10.1002/ eat. 23004.

Vainik, U., García-García, I., \& Dagher, A. (2019). Uncontrolled eating: A unifying heritable trait linked with obesity, overeating, personality and the brain. European Journal of Neuroscience, 50(3), 2430-2445. https://doi.org/10.1111/ejn.14352.

Vainik, U., Neseliler, S., Konstabel, K., Fellows, L. K., \& Dagher, A. (2015). Eating traits questionnaires as a continuum of a single concept. Uncontrolled eating. Appetite. https://doi.org/10.1016/j.appet.2015.03.004.

Voon, V., Morris, L. S., Irvine, M. a, Ruck, C., Worbe, Y., Derbyshire, K., Rankov, V., Schreiber, L. R., Odlaug, B. L., Harrison, N. a, Wood, J., Robbins, T. W., Bullmore, E. T., \& Grant, J. E. (2014). Risk Taking in Disorders of Natural and Drug Rewards: Neural Correlates and Effects of Probability, Valence and Magnitude. Neuropsychopharmacology : Official Publication of the American College of Neuropsychopharmacology, 1-9. https://doi.org/10.1038/npp.2014.242.

Watson, T. D., \& Garvey, K. T. (2013). Neurocognitive correlates of processing foodrelated stimuli in a Go/No-go paradigm. Appetite, 71, 40-47. https://doi.org/ 10.1016/j.appet.2013.07.007.

Wu, M., Giel, K. E., Skunde, M., Schag, K., Rudofsky, G., De Zwaan, M., Zipfel, S. Herzog, W., \& Friederich, H. C. (2013). Inhibitory control and decision making under risk in bulimia nervosa and binge-eating disorder. International Journal of Eating Disorders, 46(7), 721-728. https://doi.org/10.1002/eat.22143.

Yang, Y., Shields, G. S., Guo, C., \& Liu, Y. (2018). Executive function performance in obesity and overweight individuals: A meta-analysis and review. Neuroscience and Biobehavioral Reviews, 84, 225-244. https://doi.org/10.1016/j. neubiorev.2017.11.020.

Yang, Y., Shields, G. S., Wu, Q., Liu, Y., Chen, H., \& Guo, C. (2019). Cognitive training on eating behaviour and weight loss: A meta-analysis and systematic review. Obesity Reviews, 20(11), 1628-1641. https://doi.org/10.1111/obr.12916. 